\title{
LA CONCORDIA ENTRE FILOSOFÍA Y RELIGIÓN EN EL FASL AL-MAQÂL DE AVERROES (*)
}

\author{
Idoia MAIZA OZCOIDI \\ Departamento de Filosofia. UNED
}

\section{Puesto de Averroes en el pensamiento medieval}

Con el último y más célebre de los filósofos andaluces, el cordobés Abû- 1 Walîd Ibn Rušd (1126-1198), renace en Europa el naturalismo aristotélico y el Islam es explicado desde una perspectiva racionalista. Occidente conocería a Aristóteles a través de sus ojos, sin separarse nunca su figura de la del estagirita en toda la Edad Media.

Pero Averroes no fue sólo el mejor intérprete del Corpus Aristotelicum y el más influyente aristotélico en la Edad Media y el Renacimiento. Fue también un pensador original que recreó el legado clásico griego fecundándolo con las aportaciones de la cultura islámica. Considerar a Averroes como el comentarista por excelencia de Aristóteles es un tópico de la historiografía filosófica que oculta su principal aportación: hacer filosofía sin supuestos, sin aceptar más magisterio que el de la estricta razón.

Como buen musulmán Averroes no ignoró la fe islámica, pero dio a Dios lo que es de Dios, la teología, y a la sabiduría terrenal lo suyo, la filosofía. El mérito de Averroes consistió en la desneoplatonización del aristotelismo medieval, al postular la posibilidad de una filosofia pura, independiente de todo prejuicio

(*) Comunicación presentada en el Simposi Internacional de Filosofla de l'Edat Mitjana, „El pensament antropologic medieval en els àmbits islàmic, hebreu i cristiàn, Vic, Girona, 11-16 de abril de 1993.

1 Abû-l-Walid Ibn Ru'd (Averroes). Vida, obra, pensamiento, influencia, Córdoba, Publicaciones del monte de Piedad y Caja de Ahorros de Córdoba, 1986, p. 12. 
teológico. Como ha señalado M. Cruz Hernández ${ }^{1}$, Averroes sigue a Aristóteles porque "no quiso quedarse ni en la vía extrema escéptica y antifilosófica de los teólogos mutakallimîn a’saries, ni en la vía aviceniana de mezcla de filosofía y teología, sino en la vía de la estricta filosofía como sabiduría humana, que no es el saber total y absoluto que sólo a Dios compete, sino la búsqueda racional de la verdad». Averroes se nos presenta así como aquél genial innovador que, en pleno siglo XII, planteó la posibilidad de ser científico y filósofo, y, además, creyente.

\section{Revelación, filosofía y kalâm}

El problema del conflicto o de la armonía entre el ámbito religioso y el filosófico o científico se presenta bastante pronto en la comunidad musulmana, apenas la doctrina metafísica y teológica contenida en el Corán tuvo que enfrentarse con la teoría de la Grecia pagana. Tras ese contacto con la cultura griega los teólogos musulmanes encontraron útil servirse de la filosofía para robustecer sus argumentaciones y para dar un fundamento más sólido a sus investigaciones sobre lo divino, sobre la naturaleza y sobre la sociedad.

No debemos olvidar que el Islam es una religión muy racionalista. Su concepción de las exigencias y límites de la razón hacen de ella una especie de razón raciocinante, con tendencia agnóstica en lo referente al misterio de Dios, y con tendencia rigorista en la aplicación de los principios de contradicción y evidencia en lo referente a su obra creada. No debe, por tanto, extrañarnos que los pensadores del Islam, que en su mayoría fueron, además de filósofos, teólogos, intentaran ya desde muy pronto razonar su propio credo utilizando para ello la metafísica y la lógica griegas.

No obstante, las soluciones a los problemas planteados como el que nos ocupa, no fueron únicas, ni fáciles. Por ejemplo, entre las cuestiones debatidas entre las numerosas escuelas de pensamiento figuran, en primer lugar, las referentes a la naturaleza del 'aql (intelecto, inteligencia, razón) ${ }^{2}$. A cada posición doctrinal corresponde una escuela, por lo que nos encontramos ante un gran abanico de opiniones que sugieren un clima de efervescencia especulativa antes del siglo X. Como ha subrayado M. Arkoun ${ }^{3}$, no se ha insistido lo suficiente so-

2 Cfr. L. GaRdet y M. ANAwati, Introduction d la théologie musulmane. Essai de théologie comparée, París, Vrin, 3.a ed, 1981, pp. 347-364.

3 Pour une critique de la raison islamique, Paris, Maisonneuve et Larose, 1984, p. 88. 
bre el papel positivo de todas las razones concurrentes para valorar la noción de una razón islámica plural y dialéctica, correspondiente a una realidad sociológica, histórica e incluso geográfica. El cuadro polémico e ideológico en el cual se discutieron la mayoría de los temas es reflejado por las soluciones formalmente opuestas del kalam, teología islámica, y de la falsafa, filosofía islámica.

Llegados a este punto parece imprescindible una precisión. Existe la costumbre de considerar a la falsafa como la filosofía del Islam y al kalàm como su teología. Sin embargo, hay que tener en cuenta que la significación de la teología en el cristianismo y del kalàm en el Islam no es modo alguno la misma. Por otra parte, de la misma manera que los falasifa integraron desde sus comienzos todas las cuestiones teológicas planteadas por el Islam, el kalàm, aunque en un principio no supiera proveerse de un utillaje racional suficiente, tendió progresivamente a integrar toda la filosofía que estimó podía hacer suya tras sus contactos con la falsafa. No lo hizo, desde luego, distinguiendo la filosofia de la teología, sino que toda la filosofía que heredaba y transformaba según sus necesidades pasó a ser kalâm. Por esta razón, ulas relaciones entre el kalam y la falsafa no podrían ser comprendidas como las de una teología y una filosofía. Se trata más bien de dos líneas de pensamiento divergentes, entendiendo cada una en su plano bastarse enteramente por sí misma" 4 .

La aparición del kalám significó en el Islam el intento de racionalizar la fe. Ibn Khaldûn en su Muqaddima definió esta ciencia como sigue: "La ciencia del kalam es una ciencia que provee los medios para probar los dogmas de la fe a través de argumentos racionales, y para refutar a los innovadores que, en lo que respecta a las creencias, se separan de la doctrina seguida por los antiguos y por las gentes de tradición. La esencia misma de estos dogmas es la unicidad de Dios" 5. También Al-Fârâbî lo había definido como el arte que tiene por fin "defender los dogmas exigidos popr el fundador de la religión y condenar lo que se oponga a ellos por medio de razonamientos" 6 .

La falsafa es sin ninguna duda una filosofía de inspiración profundamente helenística. Dominantemente neoplatónica (sin excluir a Aristóteles) en AlFârâbî y Avicena y fielmente aristotélica en Averroes. Tampoco hay duda de que

4 L. GARDET y M. ANAWATI, Introduction à la théologie musulmane, ed. cit., p. 319.

5 Cit. en M. ARKoun, Pour une critique de la raison islamique, op. cit., p. 309.

6 Catálogo de las ciencias, trad. de González Palencia, Madrid-Granada, CSIC, 1953, p. 3. 
se trata de una auténtica filosofía musulmana ${ }^{7}$. Si bien el concepto de filosofía islámica puede ser un concepto ambiguo y polivalente, no por ello no pueden establecerse algunos puntos firmes: en primer lugar, la filosofía islámica, sea o no lícito utilizar esta expresión, está vinculada al doble hito de la experiencia religiosa coránica y al cuadro político ideológico de la sociedad en que se desarrolla. En segundo lugar, el acercamiento filosófico desencadenó en algunos pensadores islámicos importantes conflictos morales —caso típico es el de Algazel ${ }^{8}$ - por los cuales la relación entre revelación y filosofía en hombres como Averroes - caso opuesto al de Algazel - se resuelve en la necesidad de solucionar el problema en clave conciliativa, conservando por una parte la fidelidad 'ortodoxa' a los dogmas y a los comportamientos, y, por otra, reivindicando la dignidad y autonomía de la investigación científica.

\section{El Fasl al-Maqâl en su contexto histórico: el mâlikismo y la reforma almohade}

El málikismo y la incidencia del poder almohade en la cultura andalusí son dos problemas directamente relacionados con Averroes y su obra. Como ha subrayado M. Arkoun 9, «de todas las formas de pensamiento introducidas en el Occidente musulmán, el pensamiento ético-jurídico maliké fue el más influyente, el más ampliamente difundido y el más duradero. Este dato sociocultural ha sido tan constante, que desde la introducción oficial del málikismo en el año 796, ha condicionado prácticamente toda la actividad intelectual en el Occidente musulmán hasta la intervención de la modernidad».

El málikismo es una de las cuatro escuelas jurídicas del Islam sunní. Bajo el férreo control de los juristas malikis, el mundo intelectual español y maghrebí estaba, al menos oficialmente, cerrado a toda posible sugestión filosófica y ajeno al ejercicio de la libre especulación, sobre todo en el campo de la dogmáti-

7 L. GaRDET y M. ANawati discrepan de este parecer. En su opinión, su filosofla no fue una filosofia musulmana. Tampoco una filosofía árabe como proponía Gilson, sino, más bien una filosofía de inspiración esencialmente platónico-aristotélica, de expresión árabe y de influencia musulmana. Cfr. op. cit., p. 321.

8 Cfr. O. Leaman, «Le seduzioni e i mali della filosofia secondo Al-Ghazaliw, en M. CAMPANINI, L'Intelligenza della fede. Filosofia e religione in Averroè e nell'averroismo, Bergamo, Pierluigi Lubrina (ed.), 1989, pp. 129-132.

9 Op. cit., p. 320. 
ca religiosa. Tenemos, por tanto, derecho a preguntarnos cómo Ibn Tufayl o Averroes pudieron practicar la filosofía en una sociedad sometida a la ideología almohade, cuya doctrina prescribía la sumisión al Mahdî, intérprete autorizado de la ley religiosa. La cuestión se plantea tanto más, cuanto que la filosofía no había conocido todavía en el siglo XI más que una existencia limitada y sobre todo precaria en Al-Andalus.

La biografía de estos pensadores muestra que trabajaron bajo el temor de los jurisconsultos (fuqahâ') y la necesidad de cambiar las posiciones 'ortodoxas'. Esto explica la desaparición de muchas obras y, sobre todo, el olvido reservado a su enseñanza tras la muerte de Averroes. Sólo bajo los reinados de Abû Yáqûb Yûsuf (1163-1185) y de su hijo Abû Yûsuf (1185-1190) la filosofía conoció una coyuntura favorable en el Occidente musulmán. En los testimonios de al-Marrâkusî, el historiador de los almohades, aparece claramente que el apoyo del príncipe es una de las condiciones necesarias para el ejercicio de la filosofia. A este respecto, Ibn Tufayl tuvo más suerte que Averroes, pues conservó la amistad del califa hasta su muerte en 1185 . No le pasó lo mismo a Averroes, que poco antes de morir fue víctima de una cábala montada, sin duda, por los fuqahà' de Córdoba. El episodio es significativo del renadío de autoridad del mâlikismo bajo Abû Yáqûb Yûsuf, que había secretamente renunciado a la doctrina almohade.

Como ha señalado A. Martínez Lorca ${ }^{10}$, prejuicios de diverso origen, mezclados con tópicos inconsistentes, han oscurecido durante mucho tiempo la mayoría de los análisis sobre el málikismo andalusí. Pero no es menos cierto que la celebridad de Ibn Tufayl o la importancia excepcional de las obras de Averroes y de Maimónides nos hagan hoy olvidar el clima inquisitorial en el cual estos espíritus trabajaron.

Teniendo en cuenta que la restauración de la verdadera filosofía está presente en todas las obras de creación original de Averroes, y considerada la personalidad de Abû Yaqûb Yûsuf y su culto por la filosofía, es natural que Averroes se sintiese estimulado a escribir alguna obra apologética de la filosofía contra el oscurantismo y la negativa, sobre todo de los juristas malikts, para utilizar la investigación racional también en el campo teológico. Este es el fin del Fasl al-Maqâl, que representa el principal momento de la reflexión de Averroes sobre el problema de las relaciones entre filosofía y religión: p. 68 .

10 Cfr. Introducción a Ensayos sobre la filosofia en Al-Andalus, Barcelona, Anthropos, 1990, 
En el Fasl Averroes intentó, ante todo, ofrecer una respuesta legal a la condena de la filosofía pronunciada por Algazel y compartida por los teólogos as̆aries. El público al que la obra se dirige estaba, sin embargo, presumiblemente compuesto por andalusíes educados en la tradición legal málikí. Así, mientras que en él se evidencia un claro antagonismo respecto a la teología ăsari, el opúsculo se dirige a sus lectores con tono persuasivo, aplicando argumentos legales a las cuestiones filosóficas y efectuando paralelismos entre la filosofia y la jurisprudencia ${ }^{11}$.

El Fasl resulta, por tanto, si no adecuado, al menos no contrastado con la política almohade. De hecho se ha interpretado como la puesta en práctica de las teorias religiosas almohades y como la obra en donde más claramente queda reflejada la huella de tal doctrina. Esto es cuanto intenta demostrar Rosenthal, subrayando cómo Averroes, pese a que tuvo que actuar con cierta cautela, escribió cuanto efectivamente pensó; "la apasionada defensa averroísta de la filosofía y de los filósofos contra Algazel, y en particular contra los mutakallimûn... indica claramente que Averroes estaba convencido de verdad del hecho de que las intenciones de la filosofia eran idénticas a las de la sharîa (ley religiosa)» 12 .

La filosofía, dada su complejidad intrínseca, queda reservada para los filósofos, mientras que la religión se dirige a todos, filósofos y no filósofos. El conocimiento abstracto del Corán sólo puede ser alcanzado y plenamente comprendido por los filósofos, que se sirven del razonamiento demostrativo. $\mathrm{La}$ masa debe aceptar el sentido literal del texto, las enseñanzas retóricas y poéticas.

Averroes en el Fasl establece una distinción entre la teología, asequible a unos pocos, y la religión, obligada a todos los hombres. En realidad, la religión es la filosofía hecha asequible al vulgo bajo velos simbólicos, para que éste pueda acercarse a la verdad, pues "Dios se dirige siempre a todos los hombres del modo como estos puedan comprender mejor, para tocar sus corazones" (Corán 16, 26). Por eso, para Averroes, "aunque todos los musulmanes estemos convencidos de que nuestra Ley divina es la verdad y que ella misma es la que nos incita y llama a esta felicidad de conocer a Dios... y sus criaturas, este conocimiento debe estar en proporción al grado de inteligencia de los distintos hom-

11 G. Hourani, Averroes on the harmony of religion and philosophy, Londres, 1976, p. 17.

12 "Averroè intellettuale musulmano", en M. CAMPANINI, L'Intelligenza della fede, ed. cit., pp. 133-134. 
bres» ${ }^{13}$. La diferencia de capacidad entre los hombres hace necesaria la interpretación de los textos oscuros, y en esa interpretación (ta'wî́) los filósofos verifican la concordia entre revelación y razón, entre religión y filosofia. El común de los hombres disfruta de este acuerdo sin conocerlo. No obstante, Averroes insiste en que hay que respetar su situación y no desvelarle esas interpretaciones que, por no estar en condiciones de comprenderlas, suscitarían las sectas y confusión entre los fieles.

El filósofo, único que sabe penetrar con argumentos demostrativos en los más íntimos significados de la Ley, cumple así una importante función política en el Estado musulmán. Por eso Averroes, en tanto que musulmán, se preocupó por la pureza del Islam y la ortodoxia de la masa, llegando incluso a invocar la intervención del poder secular del gobierno almohade ${ }^{14}$, dirigiéndose al califa, para que se prohibiera a los mutakallimûn propagar el sentido esotérico de la Escritura. De aquí su hostilidad hacia los mutakallimûn que usan argumentos dialécticos, insuficientes, y fuente de corrupción para la masa. De aquí también su insistencia sobre el hecho de que los filosofos - cuya pretensión de ser los únicos legítimos intérpretes del contenido teórico de la Ley viene justificada en el Fast- no deben de ningún modo divulgar la verdad oscura a la masa. "Quien comunica la interpretación alegórica al vulgo, incapaz de comprenderla, corrompe la revelación y desvía de ella a los hombres, por lo cual ha de ser tachado de infiel" 15 . Como se ve por estas palabras, para Averroes el peligro para la fe no podía venir nunca de los sabios o filósofos, sino de los divulgadores del Kalâm, que con su manera de comportarse habían desorientado y enfrentado a los fieles entre sí.

La conclusión de Averroes de que la tarea del filósofo es sacar a la luz la verdad oculta en la Ley revelada y la verdad especulativa, obtenida demostrativamente, presenta un perfecto acuerdo con la doctrina almohade, formulada por Ibn Tumart, y puesta a la base del Estado por el fundador y primer califa de la dinastía. Ibn Tumart reclamaba la fe en la unicidad de Dios, integrada en el complementario conocimiento de la sharî̀a y de sus ensenanzas. Excluía la posibilidad de la opinión subjetiva, y recomendaba el uso del qiyas sharî’a (la deducción legal por analogía). En su intento de explicar la sharîa, Averroes había, ciertamente, concordado con este presupuesto, pero en tanto que filósofo, in-

\footnotetext{
13 Fash ed. cit., p. 161

14 Cfr., ibid., p. 186.

15 Ibid., p. 164.
} 
sistió en el qiyás 'aqlî (la deducción lógica por analogia) que Ibn Tumart había excluido de los ûsúl al-figh, los principios de la jurisprudencia. Oponiéndose al kalam desarrollado por Al-A šarí y su escuela, Averroes debía por fuerza oponerse también a Ibn Tumart en las cuestiones teológicas. Ahora bien, podía sentirse seguro en tanto que sus dos señores, el segundo y tercer califas almohades, como ya hemos visto, se habían alejado de Ibn Tumart en este punto.

En el Fasl Averroes aplaude la reforma almohade con las siguientes palabras:

Muchos de esos males y extraviados métodos [se refiere a la enemistad recíproca de las sectas] disipáronse hace tiempo, porque Dios así lo dispuso, principalmente con el método que yo indico, el cual trajo consigo grandes bienes [se refiere al gobierno de los almohades], sobre todo para aquellos que empleando el procedimiento de la especulación racional, anhelan sinceramente conocer la verdad. $Y$ es que ese método convida al vulgo, ávido de conocer a Dios, a caminar por una senda media, en la que se ve exento de la humillación propia de los que creen sin razones de ninguna especie y de la sofistería propia de los teólogos, los mutakallimûn, sin dejar de sugerir a los hombres elegidos la necesidad de inquirir por la razón los fundamentos de la revelación ${ }^{16}$.

Más allá de un posible intento de elogiar a los soberanos almohades, este pasaje sugiere, como ha señalado Rosenthal ${ }^{17}$, que los almohades se habían afanado en difundir entre la masa los principios fundamentales de la Ley, al contrario que sus predecesores almorávides, que tendieron a mantenerla en la ignorancia. El pasaje indica, además, que los almohades no pusieron obstáculos a hombres como Ibn Tufayl y Averroes, empeñados en extraer el sentido exotérico de la Ley por medio de argumentos demostrativos.

\section{4. el racionalismo de Averroes}

La afirmación central contenida en el Fasly que resulta el fundamento imprescindible para todo juicio sobre la obra, es que «el razonamiento filosófico no nos conducirá a conclusión alguna contraria a lo que está consignado en la revelación divina, porque la verdad no puede contradecir a la verdad, sino armonizarse con ella y servirle de testimonio confirmativos ${ }^{18}$. Este es el argumento cen-

\footnotetext{
16 Ibid., pp. 199-200.

17 Art. cit., p. 138.

18 Fash ed. cit., p. 161.
} 
tral de Averroes en defensa de la concordia entre revelación y filosofía: la verdad revelada es cierta y no puede conducir a la falsedad. Si la misma revelación nos invita a razonar, el esfuerzo racional tampoco puede conducirnos al error.

Esto significa que para Averroes no existen dos verdades. Si por un lado existiese el pensamiento filosófico y por otro el religioso, la conclusión sería necesariamente el escepticismo sobre una de las partes, actitud que tan insistentemente criticó Averroes en Algazel y los mutakallimûn. Más que de dos verdades se trata de dos territorios, de dos planos de visión que convergen en una misma verdad.

La interpretación del pensamiento de Averroes, y en particular de la relación entre filosofía y religión, ha estado condicionada por los escritos de Gauthier, que lo acusa de un racionalismo absoluto. Este autor entiende el racionalismo en un sentido bastante tradicional: racionalista sería aquél que privilegia el método especulativo sobre todo método de conocimiento, y considera el fruto de la actividad racional más cierto y satisfactorio que cualquier otro género de actividad epistemológica, y en particular, que la religión. Gauthier insiste en el hecho de que para Averroes la religión es, sobre todo, útil, mientras que la filosofía constituye la única ciencia verdaderamente rigurosa ${ }^{19}$.

El análisis de Gauthier, aceptado probablemente sin un conocimiento directo de la obra de Averroes, ha conducido a un forcejeo, si no a un malentendido en la historia de la crítica. E. Gilson, por ejemplo, sostiene que para Averroes "el conocimiento filosófico es el tipo de saber perfecto, del cual la fe sólo es una útil pero chabacana aproximación" 20 . Es evidente que este razonamiento se parece al de aquellos adversarios medievales de Averroes, que lo acusaban de considerar la religión menos verdadera que la filosofía. El hecho es que, como ha subrayado con acierto M. Campanini, «interpretaciones como las de Gauthier, repetidas por estudiosos como Gilson, se fundan en el grave equívoco de confundir la verdad con la certeza. Si la primera es igual para todos (la verdad no puede contradecir a la verdad), el grado de certeza es proporcional a los instrumentos cognitivos adoptados (la filosofía, por ejemplo, proporciona más certeza que la dialéctica) y a las aptitudes psicobiológicas de los individuos" 21 .

19 La theorie d'Ibn Rochd (Averroès) sur les rapports de la religion et de la philosophie, París, Vrin, 1909, p. 41.

20 Dante e la filosofia, cit. en M. CAMPANINI, L'Intelligenza della fede, op. cit., p. 26.

21 L'Intelligenza della fede, op. cit., pp. 26-27. 
A menudo, para probar el pretendido racionalismo de Averroes, se da como ejemplo la primacía concedida por él a la filosofía sobre la religión. Pero, en realidad, se trata de otra cosa. Ya ha quedado suficientemente claro que en el Fasl es rechazable la idea de que para Averroes la religión sea apenas una aproximación a la verdad. Lo que tiene es un lenguaje distinto al filosófico, y dirigido a una categoría sociocultural diversa a la de los filósofos. La opinión de M. Allard sobre este punto es concluyente:

Averroes da primacía, no a tales verdades filosóficas sobre tales verdades religiosas, sino a la expresión filosófica y más rigurosamente racional de una verdad, sobre la expresión religiosa de la misma verdad. Su objetivo no es denigrar al Corán haciendo resaltar la debilidad de sus argumentaciones, sino reforzar la autoridad del Libro santo por medio de la prueba apodíctica ${ }^{22}$.

Además, Averroes es categórico al sostener que los principios de la religión son todos verdaderos y que nadie, filósofo o no, puede dudar de su verdad. El tasdîq es, en efecto, uno de los términos claves del Fasl e indica que todos los hombres tienen que adherirse a la verdad, según su capacidad.

Sí es posible, como ha observado M. Campanini, que existiese en la mente de Averroes una contradicción entre fideísmo y racionalidad. Pero tal contradicción no pone en discusión la fundamental convicción de la unicidad de la verdad. Tal diferencia la resuelve el pensador musulmán insistiendo en la diferenciación social y cultural, que hace de algunos hombres sujetos más apropiados que otros para el estudio de la filosofía 23 .

El singular destino de la incomprensión de Averroes, que muchos pensadores cristianos de la Edad Media consideraron ateo, emerge con mayor evidencia aún si se procede al análisis del Tabâfut al-tahâfut o La Destrucción de la destrucción, y en un segundo plano a los comentarios, obras que, a diferencia del Fasl fueron conocidas en Occidente. Por lo que respecta al problema de las relaciones entre fe y razón, en el Tahâfut aparecen retomadas y confirmadas las argumentaciones del Fasl. Averroes no deja de asumir comprometidamente los problemas centrales de la teología en clave eminentemente ortodoxa. Por ser muchos los elementos fideístas contenidos en ésta y sus otras obras, por su referencia constante al texto coránico para probar sus asertos y por la innegable

22 «Linguaggio della filosofia e linguaggio della religione», art. cit., pp. 156-157.

23 L'Intelligenza della fede, op. cit., pp. 25-26. 
sinceridad de todo su pensamiento, parece obligado concluir que Averroes no fue racionalista. Sin embargo, nadie debería dudar a estas alturas que la cuestión del racionalismo de Averroes es, ante todo, una cuestión terminológica. «Si 'racionalismo' quiere decir negar una dimensión trascendente del saber o tener la certeza de que más allá de las conclusiones de la razón no hay conocimiento, Averroes ciertamente no fue racionalista. Si entendemos, por el contrario, el término 'racionalismo' como la voluntad de no contravenir las conclusiones que se extraen por necesidad de algunos principios presupuestos, entonces sin lugar a dudas Averroes fue racionalista. Insistió, de hecho, no sólo en el orden necesario de la ciencia, sino que afirmó sin ambigüedad que con el término de filosofía se entiende el examen teorético de las cosas conforme a la naturaleza de la demostración apodíctica. Toda epistemología tiene reglas y éstas deben ser respetadas. Tal es el núcleo del racionalismo de Averroes» 24 .

24 Ibid., p. 37. 